\title{
BMJ Open Effect of antenatal dietary myo-inositol supplementation on the incidence of gestational diabetes mellitus and fetal outcome: protocol for a double-blind randomised controlled trial
}

\author{
Ibrahim Ibrahim (10 , ${ }^{1}$ Hala Abdullahi, ${ }^{2}$ Yassin Fagier, ${ }^{2}$ Osman Ortashi, ${ }^{2}$ \\ Annalisa Terranegra, ${ }^{3}$ Gbemisola Okunoye ${ }^{2}$
}

To cite: Ibrahim I, Abdullahi $\mathrm{H}$ Fagier $\mathrm{Y}$, et al. Effect of antenatal dietary myoinositol supplementation on the incidence of gestational diabetes mellitus and fetal outcome: protocol for a double-blind randomised controlled trial. BMJ Open 2022;12:e055314. doi:10.1136/ bmjopen-2021-055314

- Prepublication history and additional supplemental material for this paper are available online. To view these files, please visit the journal online (http://dx.doi.org/10.1136/ bmjopen-2021-055314).

Received 11 July 2021 Accepted 13 December 2021

Check for updates

(c) Author(s) (or their employer(s)) 2022. Re-use permitted under CC BY-NC. No commercial re-use. See rights and permissions. Published by BMJ.

${ }^{1}$ Department of Endocrinology, Sidra Medicine, Doha, Qatar ${ }^{2}$ Department of Obstetrics, Sidra Medicine, Doha, Qatar

${ }^{3}$ Research Department, Sidra Medicine, Doha, Qatar

Correspondence to Dr Ibrahim Ibrahim; Ibrahim2002@doctors.org.uk

\section{ABSTRACT}

Introduction Gestational diabetes mellitus (GDM) affects $23.6 \%$ of Qatari women and is associated with maternal and perinatal morbidity and long-term risk of developing type 2 diabetes. A number of challenges exist with current interventions, including non-compliance with dietary advice, the reluctance of mothers to ingest metformin tablets or use insulin injections. These challenges highlight the importance of pursuing evidence-based prevention strategies. Myo-inositol is readily available as an US Food and Drug Administration-approved food supplement with emerging but limited evidence suggesting it may be beneficial in reducing the incidence of GDM. Further studies, such as this one, from different ethnic contexts and with differing risk factors, are urgently needed to assess myo-inositol effects on maternal and neonatal outcomes.

Methods and analysis This study is a prospective, randomised, double-blinded, placebo controlled clinical trial to either myo-inositol supplementation or placebo. We plan to enrol 640 pregnant women attending antenatal care at Sidra Medicine, Doha, Qatar, 320 in each arm. All participants will complete at least 12 weeks of supplementation prior to undertaking the Oral Glucose Tolerance Test at 24-28 weeks. The daily use of the trial supplementation will continue until the end of pregnancy. All outcome measures will be collected from the electronic medical records.

Ethics and dissemination Ethical approval for the study was obtained on 12 April 2021 from Sidra Medicine (IRB number 1538656). Results of the primary trial outcome and secondary endpoints will be submitted for publication in a peer-reviewed journal.

Trial registration number Prospectively registered on 26 May 2021. Registration number ISRCTN16448440 (ISRCTN registry).

\section{INTRODUCTION}

Gestational diabetes mellitus (GDM) is defined as any degree of glucose intolerance with onset or first recognition during pregnancy. ${ }^{1}$ Identified risk factors for GDM
Strengths and limitations of this study

- To our knowledge, this is the first large Randomised Controlled Trial (RCT) in an ethnic population with a high prevalence of gestational diabetes mellitus to test the effectiveness of myo-inositol supplementation.

- The early screening of diabetes in pregnancy used in Qatar ensures that women with pre-existing diabetes are excluded before enrolment.

- The duration of myo-inositol supplementation prior to Oral Glucose Tolerance Test is variable, between 12 and 20 weeks and this is a potential limitation of the study.

- The study may not have enough power to address some of the secondary outcome measures.

include maternal age, maternal body mass index, ethnic background, family history and history of GDM. ${ }^{1}$ Globally, the International Diabetes Federation estimates that $14 \%$ of all deliveries are affected by GDM. ${ }^{2}$ Diabetes is a significant public health issue in Qatar with increasing prevalence over the years. A recent study from Qatar showed that the prevalence of GDM among Qatari women was $23.6 \% .^{3} \mathrm{GDM}$ is a major pregnancy complication associated with both maternal and perinatal morbidity and long-term risk of the development of type 2 diabetes (T2DM). The Hyperglycemia and Adverse Pregnancy Outcomes (HAPO) study, a multicentric, observational study, evaluated the relationship between maternal HAPO and identified elevated maternal serum glucose level during pregnancy as a major risk factor for adverse pregnancy outcomes, increasing rates of large for gestational-age infants, fetal hyperinsulinaemia, neonatal hypoglycaemia and caesarean delivery. ${ }^{4}$ During pregnancy, GDM 
is associated with an increased risk of pre-eclampsia, preterm labour, caesarean section, macrosomia, shoulder dystocia, and a substantial increase in medical cost. ${ }^{56}$ Exposure to hyperglycaemia in pregnancy is shown to be associated with an increased risk to the offspring of abnormal glucose tolerance, T2DM, obesity and higher blood pressure. $^{78}$

In the last decade, multimodal evidence-based measures and interventions have been introduced to improve the outcome of pregnancy in women with GDM. It has also been shown that both lifestyle modifications and metformin therapy effectively delay or prevent diabetes in women with a history of GDM. ${ }^{9}$ While significant progress has been made, several challenges remain with current interventions, including non-compliance with dietary advice and mothers' reluctance to ingest metformin tablets or use insulin injections. These challenges highlight the importance of pursuing evidence-based prevention strategies.

Inositol has been proposed as a food supplement that might reduce GDM incidence in high-risk pregnant women. Myo-inositol, an isomer of inositol, is a naturally occurring sugar commonly found in cereals, corn, legumes and meat. The US Food and Drug Administration class it as a dietary supplement. It is one of the intracellular mediators of the insulin signalling pathway and correlated with insulin sensitivity in T2DM. It is an insulin-sensitising mediator, which is reported to reduce plasma glucose levels in polycystic ovary syndrome (PCOS), where it has gained increasing attention and is used for its unique property of reducing insulin resistance. Inositol isoforms were reported to improve insulin sensitivity and ovulatory function in young women affected by PCOS. ${ }^{10}$ Chiroinositol and myo-inositol are major components of the two inositol phosphoglycan mediators of insulin action. Patients with T2DM, compared with controls, have less active chiro-inositol-containing mediator fractions form and a significantly reduced chiro-inositol to myo-inositol ratio. ${ }^{11}$ Myo-inositol supplementation is shown in an RCT to reduce the incidence of GDM in pregnant women with a parent with T2DM (6\% vs $15.3 \%, \mathrm{p}=0.04$ ) as well as the incidence of the delivery of a macrocosmic fetus. ${ }^{12}$ The potential beneficial effect on improving insulin sensitivity suggests that it may be useful for preventing GDM. Although myo-inositol shows promise in preventing GDM, there is not enough evidence at this stage to support its routine use. A more recent Cochrane Database Systematic Review looked at the antenatal dietary supplementation with myo-inositol in women during pregnancy for preventing GDM, concluded that there is evidence from four trials of a potential benefit for reducing the incidence of GDM. However, there was no consensus on neonatal outcomes. The authors recommended further studies to include pregnant women of different ethnicities and varying risk factors. ${ }^{13}$ Recent systematic reviews and meta-analyses have shown that myo-inositol supplementation is associated with a significantly reduced incidence of GDM and preterm delivery. ${ }^{14} 15$
Given the ready availability of myo-inositol as a dietary supplement and its relatively low cost compared with traditional interventions for preventing GDM, exploring its potential role in reducing GDM is a much needed and timely study in a different socioeconomic and population context such as Qatar. Qatar's population is of multiethnic origin, including Qataris and non-Qatari Arabs (residents of the Middle East and North Africa Region), Asian (residents from the India subcontinent and the Philippines) and Caucasian expatriates. A large RCT in this diverse population with high GDM risk will add to the available evidence of the role of nutritional supplements to prevent GDM.

\section{METHODS/DESIGN}

\section{Aim and hypothesis}

This is a prospective, randomised, double-blind, placebocontrolled clinical trial to either myo-inositol supplementation or placebo. The study plans to enrol 640 pregnant women attending antenatal care at Sidra Medicine, with 320 pregnant women in each arm. The study overview is summarised in online supplemental figure 1.

The hypothesis for the study is that myo-inositol in pregnancy reduces the risk of developing gestational diabetes.

\section{Outcome measures}

Primary outcome:

1. The incidence of gestational diabetes in both groups Secondary outcomes:

Maternal:

1. Gestational weight gain.

2. Need for metformin or insulin therapy.

3. Mode of delivery.

4. Hypertensive disorders of pregnancy

Fetal:

1. Large for gestational age at delivery (weight $>95$ th centile for gestation).

2. Small for gestational age at delivery (weight $<10$ th centile for gestation).

3. Macrosomia (fetal weight $\geq 4000 \mathrm{~g}$ at delivery).

4. Shoulder dystocia and birth injury.

5. Polyhydramnios.

6. Neonatal Intensive Care Unite (NICU) admission for $>24$ hours.

7. Neonatal hypoglycaemia requiring intravenous glucose.

8. Preterm delivery ( $<37$ weeks gestation).

9. Transient tachypnoea of the newborn.

10. Respiratory distress syndrome (RDS).

The diagnosis of Gestational diabetes (GDM) is based on The International Association of the Diabetes and Pregnancy Study Group (IADPSG) criteria, one or more abnormal glucose values; fasting blood glucose $\geq 5.1 \mathrm{mmol} / \mathrm{L}$; 1-hour post $75 \mathrm{~g}$ Oral Glucose Tolerance Test $($ OGTT $) \geq 10.0 \mathrm{mmol} / \mathrm{L}$; and 2-hour post $75 \mathrm{~g}$ OGTT $\geq 8.5 \mathrm{mmol} / \mathrm{L}^{16}$ 
Prepregnancy weight is recorded in the initial visit based on patient self-report and is entered into the electronic medical records. In the absence of a validated prepregnancy weight, the measured weight at first hospital visit will be used with appropriate adjustment for gestation at recruitment. We will use the last recorded weight at the time of delivery to calculate total gestational weight gain corrected for gestational age at delivery.

Macrosomia is defined as birth weight $>4000 \mathrm{~g}$. Large for gestational age is defined as birth weight $>95$ th percentile, and small for gestational age is defined as birth weight $<10$ th percentile. The percentile for growth will be extracted from the built in growth charts (WHO birth to 24 months weight for age chart) within the electronic medical records system. Preterm delivery is defined as delivery $<37$ weeks' gestation.

Hypertensive disorders in pregnancy will be diagnosed based on the American College of Obstetrics and Gynaecology. ${ }^{17}$ Gestational hypertension is defined as a systolic blood pressure of $140 \mathrm{~mm} \mathrm{Hg}$ or more or a diastolic blood pressure of $90 \mathrm{~mm} \mathrm{Hg}$ or more, or both, on two occasions at least 4 hours apart after 20 weeks of gestation in a woman with a previously normal blood pressure. Women with gestational hypertension with severe range blood pressures (a systolic blood pressure of $160 \mathrm{~mm} \mathrm{Hg}$ or higher, or diastolic blood pressure of $110 \mathrm{~mm} \mathrm{Hg}$ or higher) are diagnosed with preeclampsia with severe features. Pre-eclampsia is any raised blood pressure as above with significant Proteinuria of $300 \mathrm{mg}$ or more per 24 hour urine collection or protein/creatinine ratio of $0.3 \mathrm{mg} / \mathrm{dL}$ or more or urine dipstick reading of $2+$ of protein. ${ }^{17}$

Shoulder dystocia is the use of any additional manoeuvres for the delivery of the baby after the delivery of the head, as documented by the delivering medical staff.

The diagnosis of neonatal hypoglycaemia and RDS will be extracted from the diagnosis section of the neonatal electronic medical records.

Neonatal hypoglycaemia will be diagnosed based on the Paediatric Endocrine Society and British Association of Perinatal Medicine. ${ }^{18}$ If the blood glucose is less than $45 \mathrm{mg} / \mathrm{dL}(2.5 \mathrm{mmol} / \mathrm{L})$, glucogel with a milk feed is to be given up to three times. If blood glucose remains at $25 \mathrm{mg} / \mathrm{dL}(1.4 \mathrm{mmol} / \mathrm{L})$ up to two times, then the neonate will be admitted to the Neonatal Intensive Care Unit (NICU) for intravenous dextrose. In symptomatic patients with glucose $<45 \mathrm{mg} / \mathrm{dL}(2.5 \mathrm{mmol} / \mathrm{L})$, the neonate will be admitted to NICU for intravenous dextrose. We will only include neonatal hypoglycaemia requiring intravenous glucose for this study.

RDS is coded in accordance with -ICD-10-CM diagnosis code.

Inclusion and exclusion criteria

All pregnant women booking for antenatal care at Sidra Medicine before 16 weeks of gestation will be approached to participate in the study. Comprehensive written and verbal information will be provided in both English and Arabic. This will ensure early commencement of the study supplementation or placebo with a sufficient period prior to undertaking the OGTT, thus optimising any potential effect of the intervention.

The following are the inclusion and exclusion criteria for the study:

Inclusion criteria:

1. Pregnant women booked for prenatal care at Sidra Medicine.

2. Gestational age less than 16 weeks.

3. Capacity to provide informed consent.

Exclusion criteria:

1. Pregestational diabetes/pre-existing diabetes.

2. Fasting glucose of $\geq 5.1 \mathrm{mmol} / \mathrm{L}(92 \mathrm{mg} / \mathrm{dL})$.

3. Women on steroids during pregnancy.

4. Women using metformin for any other disorder, for example, PCOS.

5. Women taking myo-inositol as part of any supplementation.

6. Cancer-not in remission.

7. Known allergy to myo-inositol

8. Women who had bariatric surgery.

9. Involvement in another interventional trial.

10. Polyhydramnios.

\section{Study participants}

Women who agree to participate will be invited to attend the research clinic. They will be provided with detailed information about the study by the principal investigator or delegated Coinvestigators, and, if agreeable, a written consent form will be obtained (online supplemental file $1)$.

An acceptance rate of $50 \%-60 \%$ is anticipated; hence, the target is to approach about 1000 women with a view to enrolling them in the study. The average obstetric antenatal booking patient rate is between 8 and 14 per day, and this translates into a projected average of 2860 patients annually, well above the recruitment target for this study. The study protocol is consistent with the principles of the Declaration of Helsinki (Institutional Review Board number 1538656), and participants will be required to provide written informed consent prior to trial enrollment. The study overview is summarised in online supplemental figure 1 .

\section{Randomisation and study schedule}

Randomisation will be performed using computergenerated numbers, which would allocate participants to either myo-inositol or Placebo arms. Both myo-inositol and placebo will have identical packaging prepared at the source and supplied through Sidra pharmacy. All research team members and research participants will be blinded to the content of the research packs. The pharmacist shall seal, randomly number the sachets according to the computer-generated scheme, and be the sole healthcare provider to access these data until the blinding is broken. Breaking blinding is possible after delivery and in cases of adverse reactions or if severe side effects are encountered. 
The myo-inositol pack will contain $2 \mathrm{~g}$ of myo-inositol in the form of a sachet. The placebo pack will contain a pharmacologically inert substrate in a similar packing to the myo-inositol sachets. Both myo-inositol and placebo sachets are to be taken twice a day.The pharmacist will provide a monthly (30 days) supply of the trial packs. All participants will complete at least 12 weeks of intervention or supplementation before undertaken OGTT. The Research nurse, blinded to the research supplements, will arrange scheduled contacts with all participants every month to arrange additional trial packs and check on compliance. These scheduled contacts will be linked to the regular antenatal clinic schedules so that participants do not have to make extra visits. All participants will have standard antenatal care as per Sidra's prenatal care pathway.

Participants will be advised to stop study supplements only when admitted in spontaneous labour, for Induction of Labour or Lower Segment Caesarean Section, whichever comes earlier. The remaining and unused sachets will be collected on admission for delivery to evaluate patient compliance. The study schedule is summarised in the online supplemental table 1 .

\section{Clinical assessment}

The OGTT will be performed at 24-28 weeks as per Sidra protocol for routine screening for GDM, and additional blood samples for C-peptide and insulin will also be taken at the same time. All participants will complete at least 12 weeks of intervention or supplementation prior to undertaking the OGTT. Women will be advised to continue using the trial packs regardless of OGTT results. Those who are diagnosed as having GDM will have standard antenatal care as per Sidra prenatal care pathway.

Data regarding pregnancy course and delivery outcome will be collected by the research nurse. Lifestyle and dietary data will be collected at each visit by a dietician using 24 hours dietary recall and questionnaires and correlated with the study outcomes (online supplemental files 2-4).

Participants who withdraw from the study or who fail to return for follow-up assessments shall continue to have data collected from their routine diabetic or obstetrics clinic visits unless they specifically withdraw consent for this. Data collected during the trial will be stored securely with appropriate data security governance in the hospital electronic system for health records as a source document.

\section{Sample size and statistical analysis}

The overall prevalence of newly detected diabetes in pregnancy in Qatar is 23.6\%. A sample size of 640 pregnant women, with 320 in each arm, is sufficient to detect a clinically significant reduction of $40 \%$ in the incidence of GDM between groups using a two-tailed z-test of proportions between two groups with $80 \%$ power and a $95 \%$ level of confidence, accounting for $20 \%$ drop out rate. This $40 \%$ reduction represents a $23.6 \%$ incidence of newly detected diabetes in pregnancy in the placebo group and a maximum of $14.2 \%$ in the myo-inositol group. All randomised subjects will be included in the analysis.

All statistical analyses will be applied using $\mathrm{R}$ and SAS V.9.4 (SAS Institute). Statistical analyses will include both descriptive (numerical and graphical) and inferential statistics. Graphical analyses, where deemed appropriate, will be included in the analyses. Frequencies and proportions will be used to summarise qualitative variables, whereas means, median, SD and quartiles will be used to summarise quantitative variables. Two sided Student's t-test and $\chi^{2}$ test will be used to evaluate the differences between continuous and categorical variables, respectively. Spearman's correlation coefficients will be estimated to determine associations between quantitative variables. Logistic regression analysis will be performed to estimate ORs and to examine the predictive effect of each factor. ORs and their 95\% CI for associated factors will be estimated, and all statistical assessments will be considered significant at $\mathrm{p}<0.05$. The statistical analyses are directed towards the assessment of the objectives of the study.

\section{Data monitoring plan}

The data-monitoring plan for this study includes the appointment of an independent expert to perform and oversee the interim analysis for the study. An interim analysis is planned at 12 months and or at $50 \%$ of enrolment, whichever comes earlier. If the interim analysis shows that the difference between the two arms reaches statistical significance, the result of the analysis and recommendation for stopping the trial will be communicated to the IRB.

Correction notice This article has been corrected since it first published. Author name 'Annalisa Terranegra' has been updated.

Contributors HA initiated the idea for the study. II, GO and HA produced the initial study design. G0 prepared the draft manuscript. All authors contributed to the manuscript review. All authors read and approved the final manuscript.

Funding The study is funded by Sidra Medicine through the competitive Internal Research Grant fund (grant number SDR200067), following the external peer review of the study. The funding arm of Sidra Medicine is not involved in the design of the study, management, analysis, interpretation of data; writing of the report; the decision to submit the report for publication or writing of the protocol manuscript.

Competing interests None declared.

Patient consent for publication Not applicable.

Provenance and peer review Not commissioned; externally peer reviewed.

Supplemental material This content has been supplied by the author(s). It has not been vetted by BMJ Publishing Group Limited (BMJ) and may not have been peer-reviewed. Any opinions or recommendations discussed are solely those of the author(s) and are not endorsed by BMJ. BMJ disclaims all liability and responsibility arising from any reliance placed on the content. Where the content includes any translated material, BMJ does not warrant the accuracy and reliability of the translations (including but not limited to local regulations, clinical guidelines, terminology, drug names and drug dosages), and is not responsible for any error and/or omissions arising from translation and adaptation or otherwise.

Open access This is an open access article distributed in accordance with the Creative Commons Attribution Non Commercial (CC BY-NC 4.0) license, which permits others to distribute, remix, adapt, build upon this work non-commercially, and license their derivative works on different terms, provided the original work is properly cited, appropriate credit is given, any changes made indicated, and the use is non-commercial. See: http://creativecommons.org/licenses/by-nc/4.0/. 
ORCID iD

Ibrahim Ibrahim http://orcid.org/0000-0001-5956-2751

\section{REFERENCES}

1 Buchanan TA, Xiang AH. Gestational diabetes mellitus. J Clin Invest 2005;115:485-91.

2 Cho NH, Shaw JE, Karuranga S, et al. IDF diabetes atlas: global estimates of diabetes prevalence for 2017 and projections for 2045. Diabetes Res Clin Pract 2018;138:271-81.

3 Bashir M, Abdel-Rahman ME, Aboulfotouh M, et al. Prevalence of newly detected diabetes in pregnancy in Qatar, using universal screening. PLoS One 2018;13:e0201247.

4 HAPO Group THSCR. Hyperglycemia and adverse pregnancy outcomes. N Engl J Med Overseas Ed 2008;358:1991-2002.

5 Farrar D. Hyperglycemia in pregnancy: prevalence, impact, and management challenges. Int J Womens Health 2016;8:519-27.

6 Dall TM, Yang W, Halder P, et al. The economic burden of elevated blood glucose levels in 2012: diagnosed and undiagnosed diabetes, gestational diabetes mellitus, and prediabetes. Diabetes Care 2014;37:3172-9.

7 Damm P, Houshmand-Oeregaard A, Kelstrup L, et al. Gestational diabetes mellitus and long-term consequences for mother and offspring: a view from Denmark. Diabetologia 2016;59:1396-9. doi:10.1007/s00125-016-3985-5

8 Tam WH, Ma RCW, Ozaki R, et al. In utero exposure to maternal hyperglycemia increases childhood cardiometabolic risk in offspring. Diabetes Care 2017;40:679-86.

9 Ratner RE, Christophi CA, Metzger BE, et al. Prevention of diabetes in women with a history of gestational diabetes: effects of metformin and lifestyle interventions. J Clin Endocrinol Metab 2008;93:4774-9. doi:10.1210/jc.2008-0772
10 Bizzarri M, Carlomagno G. Inositol: history of an effective therapy for polycystic ovary syndrome. Eur Rev Med Pharmacol Sci 2014;18:1896-903.

11 Asplin I, Galasko G, Larner J. Chiro-inositol deficiency and insulin resistance: a comparison of the chiro-inositol- and the myo-inositolcontaining insulin mediators isolated from urine, hemodialysate, and muscle of control and type II diabetic subjects. Proc Natl Acad Sci U S A 1993;90:5924-8.

12 D'Anna R, Scilipoti A, Giordano D, et al. Myo-Inositol supplementation and onset of gestational diabetes mellitus in pregnant women with a family history of type 2 diabetes: a prospective, randomized, placebo-controlled study. Diabetes Care 2013;36:854-7.

13 Crawford TJ, Crowther CA, Alsweiler J, et al. Antenatal dietary supplementation with myo-inositol in women during pregnancy for preventing gestational diabetes. Cochrane Database Syst Rev 2015;2015:CD011507.

14 Zhang H, Lv Y, Li Z, et al. The efficacy of myo-inositol supplementation to prevent gestational diabetes onset: a metaanalysis of randomized controlled trials. J Matern Fetal Neonatal Med 2019;32:2249-55.

15 Guo X, Guo S, Miao Z, et al. Myo-inositol lowers the risk of developing gestational diabetic mellitus in pregnancies: a systematic review and meta-analysis of randomized controlled trials with tria sequential analysis. J Diabetes Complications 2018;32:342-8.

16 Diagnostic Criteria and Classification of Hyperglycaemia First Detected in Pregnancy. Available: http://apps.who.int/iris/bitstream/ 10665/85975/1/WHO_NMH_MND_13.2_eng.pdf

17 HYPERTENSION. Available: https://www.acog.org/ /media/Task Force and Work Group Reports/public/HypertensioninPregnancy.pdf

18 Levene I, Wilkinson D. Identification and management of neonata hypoglycaemia in the full-term infant (British association of perinatal Medicine-Framework for practice). Arch Dis Child Educ Pract Ed 2019;104:29-32. 